Kinderliteratur im interkulturellen Prozeß 


\section{Kinderliteratur im interkulturellen Prozeß}

Studien zur allgemeinen und vergleichenden Kinderliteraturwissenschaft

Herausgegeben von Hans-Heino Ewers, Gertrud Lehnert und Emer O'Sullivan

Verlag J.B. Metzler Stuttgart - Weimar 
Die Deutsche Bibliothek - CIP-Einheitsaufnahme

Kinderliteratur im interkulturellen Prozess: Studien zur allgemeinen und vergleichenden Kinderliteraturwissenschaft/hrsg, von Hans-Heino Ewers ... - Stuttgart; Weimar: Metzler, 1994

ISBN 978-3-476-00989-0

NE: Ewers, Hans-Heino [Hrsg.]

ISBN 978-3-476-00989-0

ISBN 978-3-476-03522-6 (eBook)

DOI 10.1007/978-3-476-03522-6

Dieses Werk einschließlich aller seiner Teile ist urheberrechtlich geschützt. Jede Verwertung außerhalb der engen Grenzen des Urheberrechtsgesetzes ist ohne Zustimmung des Verlages unzulässig und strafbar. Das gilt insbesondere für Vervielfältigungen, Übersetzungen, Mikroverfilmungen und die Einspeicherung und Verarbeitung in elektronischen Systemen.

๑ 1994 Springer-Verlag GmbH Deutschland Ursprünglich erschienen bei J. B. Metzlersche Verlagsbuchhandlung und Carl Ernst Poeschel Verlag GmbH in Stuttgart 1994 
Inhalt

Vorwort ...................... VII

\section{Theorie}

Zobar Shavit

Beyond the Restrictive Frameworks of the Past: Semiotics of Children's Literature

- A New Perspective for the Study of the Field. . . . . . . . . . . . . . 3

Hans-Heino-Ewers

Theorie der Kinderliteratur zwischen Systemtheorie und Poetologie.

Eine Auseinandersetzung mit Zohar Shavit und Maria Lypp. . . . . . . . . . . 16

\section{Gertrud Lebnert}

Phantasie und Geschlechterdifferenz. Plädoyer für eine feministisch-komparatistische

Mädchenliteraturforschung. . . . . . . . . . . . . . 27

\section{Reinbert Tabbert}

Was macht erfolgreiche Kinderbücher erfolgreich?

Vorläufige Ergebnisse einer Untersuchung. . . . . . . . . . . . . . . . . 45

\section{Rezeption, Wechselwirkungen, Analogien}

\section{Göte Klingberg}

Die west-nordeuropäische Kinderliteraturregion im 19. Jahrhundert.

Einige Vergleichende Beobachtungen. . . . . . . . . . . . . . . . 65

Nitsa Ben-Ari

The Historical Novel for Youth:

In Search of National Identity Via the Adaption of a New Genre. . . . . . . . . . 72

\section{Dieter Petzold}

Die Rezeption klassischer englischsprachiger Kinderbücher in Deutschland. . . . . 78

Fürgen Martini

Überall ist Afrika, oder: Das Eigene und das Fremde. . . . . . . . . . . . . . . . 92 


\section{3. Übersetzung und Adaption}

\section{Gideon Toury}

Zielgerichtetheit einer Übersetzung, oder: Warum wurde ein deutscher

Schlaraffenland-Text so in die hebräische Kinderliteratur übertragen? . . . . . . . . 99

\section{Ruth B. Bottigheimer}

The Injunction to Work: Children's Bibles, Class Difference, and Publishing History. . . . . . . . . . . . . . . . 115

\section{Emer O'Sullivan}

Winnie-the-Poob und der erwachsene Leser: die Mehrfachadressiertheit eines kinderliterarischen Textes im Übersetzungsvergleich.

\section{Sonia Marx}

Komik in der Übersetzung. Am Beispiel der deutschen Pinocchio-Übersetzungen und -Bearbeitungen. . . . . . . . . . . . . . . . . . . . . 154

\section{Foachim Scbultz}

Für Kinder oder Erwachsene:

Luigi Malerbas Kindergeschichten in Italien und Deutschland. . . . . . . . . 172

Basmat Even-Zobar

The Female Role in Fairy Tales: 300 years of Little Red Riding Hood. . . . . . . . 181

\section{Turgay Kurultay}

Probleme und Strategien bei der kinderliterarischen Übersetzung im Rahmen der interkulturellen Kommunikation. . . . . . . . . . . . . . . . . . . . 191

\section{Neuere Tendenzen der Forschung}

\section{Fack Zipes}

Neue kritische Ansätze zur englischen und amerikanischen Kinderliteratur seit 1980:

Eine Bestandsaufnahme. . . . . . . . . . . . . . . . . . . . . . 205

\section{Isabelle Niéres}

Kinderliteratur in Frankreich. Neuere Forschungsansätze (1981-1991). . . . . . . 217 


\title{
Vorwort
}

\author{
$\gg$ Wer auf dem Wege zur Komparati- \\ stik je glaubt, er sei an seinem Ziele \\ angelangt, muß wissen, daß er von \\ diesem Weg abgekommen ist.« \\ (Erwin Koppen)
}

Der vorliegende Band präsentiert die Ergebnisse einer von der Deutschen Forschungsgemeinschaft geförderten Tagung, die im Juli 1990 als erste ihrer Art im deutschsprachigen Bereich in Bonn stattfand: »Internationale Aspekte der Kinder- und Jugendliteratur. Theorie - Übersetzung - Rezeption«. Veranstaltet wurde sie von Erwin Koppen, Inhaber des Lehrstuhls für Vergleichende Literaturwissenschaft der Universität Bonn, der sie indessen nicht mehr miterleben konnte: Wenige Wochen zuvor war er nach schwerer Krankheit im Alter von 60 Jahren gestorben. Die Komparatistik der Bundesrepublik verlor damit einen ihrer bedeutendsten Fachvertreter, einen herausragenden Gelehrten von immenser Belesenheit und einen toleranten und verständnisvollen akademischen Lehrer und Vorgesetzten. Hans-Heino Ewers, Direktor des Instituts für Jugendbuchforschung Frankfurt, der als Mitveranstalter von vornherein maßgeblich an der Konzeption beteiligt gewesen war, übernahm dankenswerterweise die Leitung und ermöglichte so die Durchführung der Bonner Tagung.

Die Innovationskraft von Erwin Koppens Begeisterung für grenzüberschreitende literarische Phänomene sowie seine Offenheit gegenüber dem Neuen, gegenüber auch dem, was nicht dem bisherigen, eingefahrenen Wissenschaftskanon entspricht, wird gerade an der Konzeption einer solchen Tagung deutlich. Als erste komparatistische Veranstaltung zur Kinder- und Jugendliteratur in Deutschland erschloß sie sowohl der Kinderliteraturforschung als auch der Komparatistik neue Arbeitsfelder und -methoden. Die Kinderund Jugendliteratur war lange kein Thema für die traditionelle Komparatistik, obgleich sie ein komparatistischer Gegenstand par excellence ist. In ihrer internationalen Vernetzung entspricht sie mehr noch als das literarische Leben schlechthin der Goetheschen Vorstellung von Weltliteratur als einem Prozeß des wechselseitigen Austauschs der Nationalliteraturen - eben dieses Verständnis von Weltliteratur ist zentral für das Selbstverständnis der Vergleichenden Literaturwissenschaft, und damit auch das Bewußtsein für das Spezifische sprachgrenzenüberschreitender literarischer Phänomene.

Im Hinblick auf ihre Verbreitung und Rezeption durch das kindliche wie erwachsene Lesepublikum ist gerade die Kinderliteratur ein Weg zur Komparatistik, wie es kaum einen besseren gibt. Kindliche LeserInnen werden gleichsam wie von selbst zu einer internationalen Literaturkonzeption erzogen, die wenig von nationaler Beschränkung weiß. Wie selbstverständlich wird die Kinder- und Jugendliteratur von ihren jugendlichen LeserInnen so rezipiert, als gebe es nur eine unteilbare Literatur und nicht viele Literaturen in vielen Sprachen. Das hat Vor- und Nachteile und liegt unter anderem am 


\section{Vorwort}

spezifisch inhaltsorientierten Leseinteresse des kinderliterarischen Publikums. Über eine Reihe anderer Gründe ist im vorliegenden Band einiges zu lesen. So hat ein gesellschaftlich dominantes Bild von den intellektuellen Fähigkeiten der kindlichen LeserInnen zur Folge, daß etwa fremdsprachige Texte, insbesondere solche aus ganz fremden Kulturkreisen, in der Adaptation für den - beispielsweise - deutschen Buchmarkt zuweilen stark verändert, vereinfacht und somit erheblich verfälscht werden, um einem sdurchschnittlichen $<$ deutschen (oder englischen oder italienischen) Kind verständlich zu sein. Die Problematik solcher Verfälschungen liegt auf der Hand und kann gerade in einer Zeit, in der das Wort von der multikulturellen Gesellschaft in aller Munde ist und zugleich die gesellschaftlichen Entwicklungen gegenläufig sind, nicht unkritisiert bleiben, ganz abgesehen davon, ob das damit verbundene Kindheitsbild nicht selbst eine ideologische Vereinfachung darstellt.

Die Rezeption der Kinderliteratur als einer einzigen, unteilbaren Literatur ist aber auch darin begründet, daß in der Öffentlichkeit ein Bewußtsein für die literarische Übersetzung als eigenes literarisches Genre weitgehend fehlt - und damit fehlt auch das Bewußtsein für die Leistung der ÜbersetzerInnen und für deren Bedeutung hinsichtlich der Rezeption der Texte. Das wird augenfällig in der Tatsache, daß in Rezensionen außerordentlich selten - und wenn, dann oberflächlich und oft ohne jede Sachkenntnis - auf die jeweilige Übersetzung eingegangen wird. Gilt dies schon für die Literatur schlechthin, so noch mehr für die Kinder- und Jugendliteratur. An genau diesem Punkt setzt die Komparatistik ein und differenziert die Vorstellung von der einen, unteilbaren Literatur, indem sie die Vielfalt und damit die Besonderheiten und Eigenarten des vielen betont.

Die Neugier auf das Fremde, auf das andere, gehört mithin ebenso zur Komparatistik wie die Erkenntnis des Spezifischen des je einzelnen Werks, der je einzelnen Nationalliteratur. Dies hat Erwin Koppen immer wieder betont und mehr noch: er hat es praktiziert und demonstriert. Er nannte die Komparatistik deswegen einmal polyglott und international, nicht universal oder integral. In einem sehr persönlichen Text spricht er von dem »lustbetonten, fast erotischen Verhältnis zu fremden Sprachen «, das jedem Komparatisten eignen müsse, damit er die jeweilige Literatur wirklich verstehen könne (und, so möchte ich hinzufügen, damit er die Übersetzungen beurteilen kann), und von der Faszination durch das Fremde, das auch das Eigene erst interessant mache, indem es es spiegele. Er schreibt von der entscheidenden Lektion, die er selbst in der Nachkriegszeit gelernt habe, daß nämlich die deutsche Literatur »erst dann anfange, interessant zu werden, wenn sie sich, in welcher Form auch immer, mit dem Ausland einläßt. « ${ }^{1}$ Diese Position läßt sich auch und gerade auf die Kinder- und Jugendliteratur übertragen. Erwin Koppen hat sich immer wieder mit Themen befaßt, die im weiteren oder engeren Sinn mit Kinder- und Jugendliteratur zu tun hatten. Es sei hier nur verwiesen auf seine Auseinandersetzung mit Otto Julius Bierbaums deutscher Bearbeitung von Collodis Pino$c c b i{ }^{2}$ sowie auf Seminare über Kindheit als literarisches Thema oder über Kinderlitera-

1 »Über Zufall, Notwendigkeit und anderes - Ein Schlängelweg zur Komparatistik«, in: Wege zur Komparatistik. Sonderheft für Horst Rüdiger zum 75. Geburtstag, hg. v. Erwin Koppen (=arcadia-Sonderheft 1983), $63-70$. 
tur und Weltliteratur. Dieses Interesse führte nicht minder als Koppens Auffassung von Komparatistik schließlich zur Veranstaltung jener Tagung, während der in theoretischen Erörterungen und anhand von Fallbeispielen genetische und typologische Beziehungen der verschiedenen Kinderliteraturen in einem dezidiert internationalen Kontext erforscht und erörtert wurden.

Mit dem vorliegenden Band wird fortgesetzt, was die Bonner Tagung in die Wege leitete: Es wurde und wird Neuland in der europäischen Komparatistik und Kinderliteraturforschung betreten. Zum einen wurde der deutschsprachigen Komparatistik die Kinder- und Jugendliteratur als ernstzunehmender Gegenstand erschlossen, zum anderen wurde die Notwendigkeit klarer komparatistischer Fragestellungen innerhalb der Kinderliteraturforschung verdeutlicht. Diese beiden Ziele können mit einer einzigen Tagung naturgemäß nicht erreicht werden, und so führt der Band in umfassender Weise fort, was damals nur ein Impuls sein konnte. Gemeinsames Anliegen aller Beiträge ist die Betonung eines strikt literaturwissenschaftlich-theoretischen Gesichtspunkts und der damit einhergehende weitgehende Verzicht auf die auch bei LiteraturwissenschaftlerInnen oft noch vorherrschende pädagogisch-didaktische, auf konkrete Anwendungsmöglichkeiten (Leseempfehlungen etc.) zielende Perspektive. Der Aufbau des Buches wurde neu konzipiert, die meisten Beiträge wurden stark überarbeitet, und einige Beiträge kamen ganz neu hinzu, um die Analysen zu vervollständigen bzw. das Spektrum der Diskussionsbeiträge zu erweitern. Die Anordnung der nun vorliegenden Aufsätze folgt also nurmehr locker der Konzeption der Tagung: Den Anfang bilden Auseinandersetzungen mit theoretischen Ansätzen der zeitgenössischen Kinder- und Jugendliteraturforschung. Genetische und typologische Beziehungen in einem größeren Rahmen stehen im Mittelpunkt des zweiten Teils; es folgen Fallbeispiele zum Problem der Übersetzung und Adaptation. Den Abschluß des Bandes bilden zwei Forschungsberichte zur Lage der anglo-amerikanischen und der französischen Kinder- und Jugendliteraturforschung.

Gertrud Lehnert

2 »Pinocchio im Reich des Simplicissimus - Otto Julius Bierbaum als Bearbeiter Collodis«, in: Stimmen der Romania. FS für Theodor W. Elwert zum 70. Geburtstag, hg. v. G. Schmidt u. M. Tietz. Wiesbaden 1980, Teil 1: Literaturen der Romania, 225 - 242. 